\section{Europe Reveals its Strengths}

\begin{abstract}
This year's Conference on Lasers and Electro-Optics/Europe - European Quantum Electronics Conference (CLEO/Europe '96 - EQEC '96) took place in Hamburg on 8 - 13 September.
\end{abstract}

Solid-state lasers were highlighted at the opening of this year's CLEO/Europe EQEC conference in Hamburg with an outstanding review by K.J. Ebeling (Ulm) of advances and prospects for semiconductor lasers. The other plenary speaker was C. Cohen Tannoudji (Paris) who discussed ultra-cold atoms before giving a fascinating insight into possible new applications. Together with S. Svanberg (Lund), he was presented with the newly instituted EPS Quantum Electronics Prize by M. Ducloy who chairs the EPS Quantum Electronics and Optics Division [EN 27 (1996) 193]. The session also included an address by O. Svelto (Milan) celebrating the 8 oth birthday of Academician Alexander P. Prokhorov. The Nobel laureate's active interest and participation in many of the sessions was clear evidence that for him the field of lasers and electro-optics has not lost any of its fascination since the very earliest days of the subject.

\section{Two Trends}

In keeping with the plenary session, many of the other 1996 CLEO/Europe EQEC presentations also demonstrated the increasing importance of advances in semiconductor diode lasers and solid-state lasers pumped by semiconductor lasers. These advances provide a common thread running through many of today's developments in laser and optical technology. The power generated by advanced solid-state lasers is an order of magnitude or so larger than the best that could be achieved only two years ago, and European laboratories seem to have particular strengths in the area. The trend was also reflected in the exhibition which accompanied the conference where 136 companies displayed their wares.

Developments in the field of quantum electronics and optics were maybe best illustrated by considering new and emerging topics. Examples of topics of this meeting that were not present in Amsterdam in 1994 were Bose-Einstein condensation, optical lattices, vertical-cavity surface emitting lasers, non-local quantum correlations, dynamics of semiconductor lasers, semiconductor cavity quantum electrodynamics, and lasing without inversion. Very striking was the natural combination of fundamental physical problems, ranging from quantum non-locality to gravity waves with applications of lasers and optics in technology and science at large.

\section{Solid-state Lasers Progress}

An invited paper by V. Ostroumov and G. Huber (Hamburg) on their generation of $1.2 \mathrm{~W}$ continuous wave (CW) in the green region of the spectrum using an intracavity frequency doubled diodepumped microchip Nd:LSB laser attracted considerable attention. Frequencydoubled diode-pumped solid-state lasers operating in the green region with outputs of several watts were also demonstrated by several companies, so we now have the first evidence that the long awaited replacement for the argon-ion technology may be on the horizon.

High-power diode-pumped Nd:YAG lasers with more than 300 watts $\mathrm{CW}$ were reviewed by A. Tunnermann (Hanover). A. Giesen et al. (Stuttgart) have developed a novel power-scaling technique involving $\mathrm{Yb}$ :YAG disks that gave average powers as high as $150 \mathrm{~W}$ (an approach that should be applicable to other solid-state lasers). The low thermal loading of $\mathrm{Yb}$-doped solidstate lasers makes these materials very interesting for high-power applications. Hence, there were reports about a large variety of different $\mathrm{Yb}$-doped solid-state lasers such as $\mathrm{Yb}$ : fluoride phosphate glass, $\mathrm{Yb}$ :S-FAP, Yb:GGG and others. A multiplexed version of a multi-rod diodepumped Nd:YAG laser presented by a group from Bern combined the ease of end-pumping with the stability and scal-

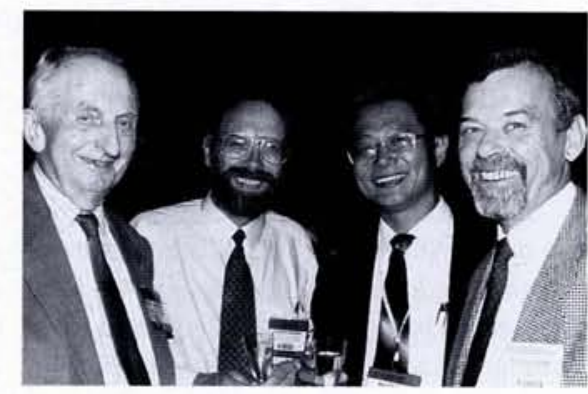

From the left, A.P. Prokhorov (Moscow), A. Ferguson (Strathcldye), B.T.H. Chai (Prando, USA), and G. Huber (Hamburg).

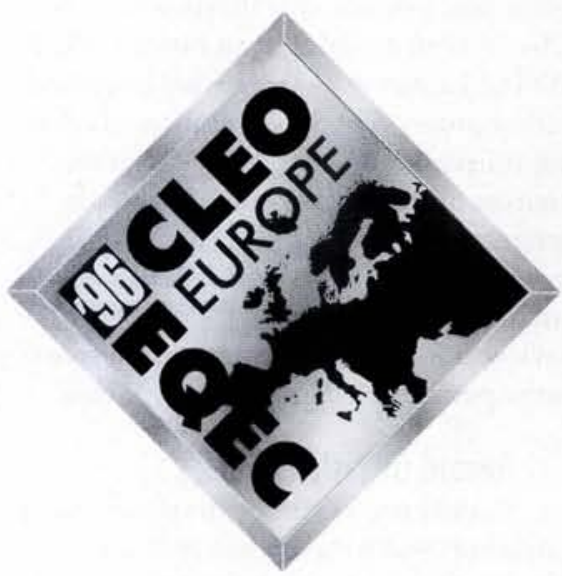

ability of symmetric multi-rod resonators to provide a laser output of $6.8 \mathrm{~W}$ and a slope efficiency of $52 \%$.

Advances in tuneable and ultra-short pulse high-power Ti sapphire lasers were also well represented. T. Brand and I. Schmidt (Berlin) reported on a 600 watt CW Nd-YAG rod laser pumped by microchannel cooled stacked diode laser arrays. They obtained 700 watt output from a double rod system, achieving a $10 \%$ wallplus efficiency.

\section{High Power Lasers Reach New Peaks}

A joint CLEO-EQEC symposium on high-peak power lasers and their applications began with an impressive invited talk by G. Mourou (Michigan, USA) entitled Ultrahigh peak power lasers: present and future. He introduced the concept of chirped-pulse amplification that has made possible terawatt lasers with high repetition rates. Today's state-of-the-art systems include a $20 \mathrm{TW}, 10 \mathrm{~Hz}$ system (Laboratoire d'Optique Applique, Paris) and a lower peak power but higher repetition rate laser with a novel amplifier from the TU Vienna. With the extremely high peak powers that can be generated nowadays it is feasible to think of table-top highenergy electron accelerators.

A novel pulse-compression technique for high-energy applications is based on a hollow fused-silica waveguide filled with noble gases. S Silvestri and O Svelto's group from Milan demonstrated compressed $10 \mathrm{fs}$ pulses with a pulse energy of $240 \mathrm{~mJ}$. The very active area of high-order harmonics generation and application was reviewed by A. L'Hullier (Lund). She described a new source of coherent short pulses to generate high harmonics in gases and discussed some of the potential spectroscopic applications. The spectroscopic applications of XUV sources in atomic and 
molecular physics were illustrated by W. Ubachs (Amsterdam) in an invited talk. B. Wellegehausen et al. (Hanover) discussed recent progress in the generation of coherent tuneable VUV-and XUV-radiation sources using excimer and Ti:sapphire lasers with low- and high order optical nonlinearities (sum-and differencefrequency mixing). P.V. Nickles (Berlin) reviewed $\mathrm{X}$-ray lasers, emphasizing shortpulse pumping schemes for soft X-rays.

\section{Record Ultrashort Pulses}

Rapid progress in the development of ultrashort pulsed lasers has continued. The unofficial news was that two European groups have broken the world record of 6 fs pulses by external pulse compression and achieved pulses as short as about $5 \mathrm{fs}$. Different techniques for measuring these ultrashort pulses in time and space were discussed.

Engineered dispersion compensation with chirped mirrors was reviewed by $\mathrm{R}$. Szipocs (Budapest). It has resulted in pulses as short as 7.5 fs directly from a Kerr lens mode-locked (KLM) Ti: sapphire laser (presented by L. Xu et al. from Vienna). Self-starting soliton mode-locked pulses as short as 10 fs using intracavity semiconductor saturable absorber mirrors were discussed by F. X. Kartner et al. (Zurich). Many applications require more compact and simpler femtosecond sources. This issue was addressed in several talks on diode-pumped $\mathrm{Cr}$ : LiSAF and $\mathrm{Cr}$ :LiSGAF lasers. Prismless KLM femtosecond $\mathrm{Cr}$ : LiSGaF lasers were reviewed by I.T. Sorokina et al. (Vienna).

\section{Gas and Metal Lasers - Efficiency Up}

Significant progress in gas and metal vapour lasers was also reported. Enhanced performance amounting to a doubling of output power of copper vapour lasers has been achieved by selectively adding trace quantities of halogen gases to the buffer gas mixture, as reported by J.A. Piper (Sydney, Australia). A copper-bromide laser providing record-breaking output power of 120 watts was described by $\mathrm{N}$. Sabotinov (Sofia). Applications of copper vapour lasers to micro-machining of materials, including the drilling and trepanning of high aspect ratio holes of 10-100 microns in diameter and several millimetres in depth in ultra hard materials such as diamond and aerospace alloys, were illustrated in a review by $\mathrm{M}$. Knowles (Abingdon). The application of $100 \mathrm{fs}$ pulses in the ablation of metals was the subject of a paper from C. Momma et al.

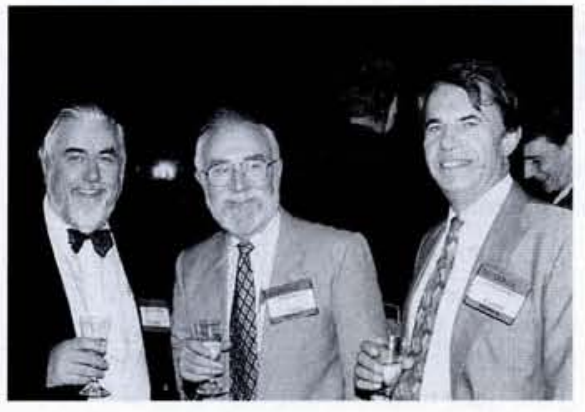

From the left, C.E. Webb (Oxford), D.R. Hall (Edinburgh) and A.R. Davies (London).

Courtesy OSA

(Hanover), in which features with scales of order a few microns were machined without any appreciable heat affected zone apparent in the surrounding material.

\section{Extending the Range}

Semiconductor lasers have seen exciting gains in performance over the last few years through their extension to both longer and shorter wavelength ranges. The available shorter wavelength range has been pushed to $410 \mathrm{~nm}$ using $\mathrm{GaN}$-based devices. For long wavelengths, a review of the $3-4 \mathrm{~mm}$ wavelength range based on III-V semiconducting alloys was given by Y. P. Yakovlev (St. Petersburg). InSb diode lasers at $5 \mathrm{~mm}$ were reported by T. Ashley (Malvern).

Surface emitting laser diodes (VCSELs) have demonstrated extremely low laser threshold currents of less than 10 $\mathrm{mA}$ and more than $50 \%$ conversion efficiency at $1 \mathrm{~mW}$ output power. At longer wavelengths, such as at $1.3 \mathrm{~mm}$ using a GaInAsP/InP VCSEL, S. Uchiyama et al. (Yokohama) reported a threshold current as low as $2.4 \mathrm{~mA}$ for $\mathrm{CW}$ operation up to $360^{\circ} \mathrm{C}$. Materials and process approaches for improved VCSELs was reviewed by R.P. Schneider et al. (Palo Alto, USA). An output of 55 watts $\mathrm{CW}$ has been obtained by C. Peyrot et al. (Paris) with a large-area $\left(>0.20 \mathrm{~cm}^{2}\right) 0.8 \mathrm{~mm}$ GaAlAs/GaAs VCSEL resulting in an optical power density of $220 \mathrm{~W} / \mathrm{cm}^{2}$. These monolithic devices are potentially useful as high-performance pump sources for solid-state lasers, replacing more expensive devices based on stacks.

The strong trend towards miniaturization by moving away from bulk scale lasers to hybrid, quasi-monolithic lasers was well represented by two sessions dedicated to diode-pumped fibre waveguide and microchip lasers. An excellent overview of microchip lasers was given by E. Molva et al. (Grenoble).
Beam Manipulation Plays a Role

Methods for combining the output of several laser diode bars and shaping their asymmetric beams into the circular beams better suited to pumping solid-state laser media was the subject of a paper by a Russian-German collaboration. As the authors acknowledged, this work builds upon the invention of the beam shaping technique introduced by W.A. Clarkson and D.C. Hanna from Southampton which was such an outstanding presentation at the 1994 CLEO/Europe in Amsterdam.

Phase conjugation represents an unique technique for adaptive control of laser radiation. It is accomplished using a remarkably wide range of nonlinear processes, each with a large choice of materials. Notable recent successes include improving the purity of stimulated Brillouin scattering (SBS) media resulting in the demonstration of diffraction-limited phase conjugation lasers providing more than $100 \mathrm{~W}$ average power. Methods for enhancing beam quality using SBS phaseconjugation were presented by H.J. Eichler et al. (Berlin), who also reported efficient and high-fidelity SBS phase-conjugation from 200 micron undoped fibres at 1.06 microns in a Nd-rod laser system. This development opens up new prospects in precision materials processing.

\section{Wide-Ranging Applications}

There were understandably many talks at the CLEO/Europe - EQEC'96 on application of lasers (optical sensors, medical and biological applications, laser material processing, lightwave communications, etc.). A symposium with speakers from LDT GmbH, Philips Research Labs and Thompson presenting the latest progress in laser projection and other large-screen TV techniques proved to be one of the most popular sessions. Also very popular was the session on surface treatment with lasers which included papers by F. Dausinger (Stuttgart) reviewing the future potential of this technique, and a paper by C. Fotakis (Heralkion) detailing the latest progress in techniques for art conservation carried out at FORTH.

\section{More Comprehensive Sensing}

In the area of laser-based remote sensing, it was noticeable that techniques are emerging for applying lidar systems to a broader range of applications. Included here were Lund University's combination of state-of-the-art IR imaging with high resolution spectroscopy, and a highly versatile lidar system from the INFM in Italy 
which has many receiver channels so that all relevant atmospheric parameters can be detected simultaneously. Meanwhile, Kayser-Threde in Munich have developed a solid-state heterodyne Doppler lidar for profiling atmospheric wind fields which is the first of its kind in Europe to be eye-safe.

Monitoring atmospheric pollution by DIAL was reviewed by M. Milton (Teddington), who showed that recent developments in laser sources have enabled effective measurements. Past and future spacebourne lidar missions were discussed by C. Werner (Wessling), where future missions have to be integrated with other remote-sensing techniques. The European Space Agency's programmes in optical remote sensing from space in preparation for new Earth-observation satellites were reviewed by E. Armandillo (Noordwijk).

\section{Optics: New Devices}

Among the many contributions addressing applications in optics mention should be made of dynamic holography for information storage and processing (reviewed by N. A. Vainos from Crete). Applications of nonlinear optics methods in molecular dynamics were summarized by S. Rosenwaks (Negev, Israel).

However, optical devices stole most of the attention. Optical switching and logic device applications were reviewed by P.W.E. Smith (Toronto, Canada) who highlighted progress in growing semiconductors for ultra-fast switching. It is now recognized that optic switch devices have the potential to improve the performance of telecommunication systems where today's electronic circuits are reaching their practical limits. In a review of optical storage, A.A. Friesem (Rehovot) reviewed the micro optic design of holographic and optical disc memories. His talk illustrated today's excitement in merging device development and innovative concepts that would have appeared unrealistic only a few years ago.

The rapid development of lightwave communications was reflected by overviews of photonic transmission and networks, active waveguides, the development of photonic components, and innovative concepts. The crucial issue of the scalability of all-optical networks was addressed by (D.J. Blumnenthal, Atlanta, USA). Also highlighted was the continued development of soliton-based technology and alloptical multiplexing.

Lightwave communications can be rendered secure using quantum encoding. Perspectives were reviewed in a sympo-

\section{CLEO/Europe-EQEC}

More than 1400 scientists, engineers and students gathered at the Congress Centrum Hamburg on 8-13 September to take part in the 1996 CLEO/Europe - EQEC conference that was sponsored jointly by the EPS (Quantum Electronics and Optics Division, the Optical Society of America (OSA) and the Lasers and Electro-Optics Society (LEOS). This was the second time that EQEC was held back-to-back with CLEO/Europe together with a technical exhibition - a formula that had turned out to be successful two years ago in Amsterdam.

The original idea for EQEC when it was held for the first time in Hanover in 1988 was to create a forum for the rapidly growing field of quantum electronics and optics so that it would be easy for students to participate. A large conference combined with CLEO naturally requires advanced facilities, which cannot be provided on the reduced budget of a meeting held in say a university. The policy of keeping a modest CLEO/Europe - EQEC registration fee for students has been very successful, for student participation in Hamburg was around $40 \%$.

For CLEO, the general chairs were D.C Hanna (Southampton) and J.-P Huignard (Orsay), and the programme chairs were C.E. Webb (Oxford) and C. Huber (Hamburg). Fifteen programme subcommittees covered different areas of research and 136 companies took part in the technical exhibition, which was sold out and many additional requests had to be turned down. For EQEC, the general chairs were E. Giacobino (Paris) and O. Poulsen (Copenhagen), with W. Ertmer (Hanover) and S. Svanberg (Lund) responsible for the programme: the local organizers was headed by G. Huber (Hanover). Contributions were selected by programme committees for spectroscopy, non-linear optical phenomena,

sium on quantum information processing, which also covered quantum computation that is potentially able to shorten extremely long calculations (e.g., factorisation). Indeed, some types of information processing technology have progressed to the point that one can now envisage exploiting quantum concepts to improve performance in several areas.

P. Zoller (Innsbruck) presented a highly interesting tutorial on quantum computing and the various aspects related to the challenging and rapidly growing field of quantum optics. A. Zeilinger, also from Innsbruck, gave an invited talk on entangled photon pairs generated in the process of parametric down conversion in a degenerate optical parametric oscillator. These and other groups are now reporting encouraging results for the building blocks a quantum computer.

Some novel optical devices were presented, including a nanometre thin-film quantum optics, optical interactions with condensed matter, physics of coherent light sources, ultrafast phenomena, lasers in chemistry, and biology and medicine.

The 1996 CLEO/Europe - EQEC had a total of nine parallel sessions, with CLEO alone having five. There were in addition six very well attended tutorials of one hour covering major research themes ranging from applications to basic physics. For EQEC, the main tutorial topics were Bose-Einstein Condensation (J.T.M.Walraven, Amsterdam) and Quantum Computing (P. Zoller, Innsbruck); other topics were clinical laser diagnostics, nearfield optical microscopy, atmosphere monitoring, and short-pulse semiconductor lasers. Short courses on miscellaneous topics of somewhat technical nature were quite instructive and well prepared, but the number of participants varied greatly from topic to topic.

There was a continuous exchange of participants between CLEO and EQEC with their 8 - 9 simultaneous parallel sessions. The sessions were well-attended and the discussions lively. Both offered an excellent opportunity for everybody to learn more about subjects outside their own area of expertise, and to obtain state-of-the-art updates over a wide variety of rapidly developing topics.

It is difficult to review a meeting of the CLEO/Europe - EQEC size in an exhaustive manner simply by highlighting contributions from among the several hundred, or even from the 50 invited talks. Readers who interested in more details are invited to consult the summaries in the Advance Programme and in the EQEC'96 Technical Digest.

The undoubted success of the 1996 CLEO/Europe - EOEC clearly owed much to the unflagging efforts of Gunter Huber and the local organizing committee. It has paved the way for another highly successful CLEO/ Europe - EQEC meeting in Glasgow in 1998.

$\mathrm{Ni}-\mathrm{NiO}-\mathrm{Ni}$ diode with integrated antennae for mixing and detection of $10 \mathrm{~mm}$ infrared laser radiation (F.K. Kneuhuhl et al., Zurich) and liquid crystal devices which act as achromatic phase elements (M. Stalder, Zurich, and M. Schadt, Basel). J. Y. Andersson et al. (Kista) discussed the possibility of using quantum-well photodetector arrays for low-cost, high resolution thermal imaging applications.

\section{Ultra Cold Atoms - Naturally}

The physics and applications of ultracold atoms naturally received considerable attention in view of the recent discovery of Bose-Einstein condensation in ultra-cold alkali metal vapours. Aside from C. Cohen-Tannoudji's plenary presentation discussing the physics of ensembles of atoms cooled to temperatures below the recoil limit, J.T.M. Walraven (Amsterdam) gave a tutorial introduction to BoseEinstein condensation. This was also the 
subject of a special symposium in which the latest results in $\mathrm{Na}$ (W. Ketterle, Cambridge, USA) and $\mathrm{Rb}$ (C. Wiemann, Boulder, USA) were presented [see EN 27 (1996) 173]. The symposium illustrated the rapid progress which is being made in the areas of excited states of condensates, nondestructive imaging and the interaction between two condensates. Proposals for an atom laser were also discussed.

In the field of cold atoms and optical lattices, the invited paper of C. Salomon (Paris) on the important observation of Bloch oscillations in a caesium lattice attracted considerable attention, as did reports concerning advances in atom interferometry. The applications of cold atoms to the nanofabrication of microstructures form a rapidly growing field of interest and was the subject of a joint CLEO - EQEC session. Nanostructures can be directly etched in silicon by laser writing, as described by M. Mullenborn et al. (Lyngby). By comparison, J.J. McClelland et al. (Gaithersburg, USA) and J. Mlynek et al. (Constance), by focusing beams of cold chromium atoms, have been able to form various patterns on substrates to achieve minimum dimensions of below only 50 $\mathrm{nm}$. The latter group also reported on etching with metastable helium atoms.

\section{Benefiting from Laser Advances}

Research in the field of optical interactions with condensed matter now ranges from single-molecule spectroscopy to clusters and nanostructure dynamics up to bulk semiconductor materials. The area has benefited greatly from recent progress in ultrafast laser technology and the development of new materials. Novel sources covering the electromagnetic spectrum from $\mathrm{THz}$ range up to the far-UV make it possible to study and manipulate elementary excitations - work that will have an enormous impact on the design of optical and optoelectronic devices.

The field was well represented at CLEO/Europe - EQEC'96, which included reports of the development of novel farinfrared sub-picosecond laser sources for studying ultrafast carrier relaxation; and of the laser-controlled picosecond creation and annihilation of carriers in semiconductors (A.P. Heberle et al., UK and Germany) that can possibly be used in ultrafast all-optical communication systems.

The Editor would like to thankW. Hogervorst (Amsterdam) and C.E. Webb (0xford) for help in compiling this report, which also made use of material reported in the Newsletter of the EPS Quantum Electronics and Optics Division (Editor: R. Corabalan) on EurophysNet at http://epswww.epfl.ch/dir/division/.

\section{Rotons in Superfluid ${ }^{4} \mathrm{He}$}

The 1996 Nobel Prize in Physics has been awarded to D.M. Lee and R.C. Richardson of Cornell University, NY, USA, and to D.D. Osheroff, Stanford University, CA, USA, for discovering superfluidity in ${ }^{3}$ He. K.H. Andersen, J. Bossy, J.C. Cook, and 0.G. Randl from the Institut LaueLangevin, Grenoble, report on recent measurements of elementary excitations in superfluid ${ }^{4} \mathrm{He}$ showing that low-temperature research still provides intriguing and challenging results.

With the liquifaction of ${ }^{4} \mathrm{He}$ in the 193 os and the discovery of superfluidity in ${ }^{4} \mathrm{He}$, it was thought that fermions such as ${ }^{3} \mathrm{He}$ followed Fermi-Dirac statistics and could not condense in the lowest energy state, so superfluidity in ${ }^{3} \mathrm{He}$ would be impossible. However, J. Bardeen, L. Cooper and R. Schrieffer proposed in their BCS theory for superconductivity that electrons in strongly cooled metals combine to form Cooper pairs, and then behave as bosons and are able to undergo Bose-Einstein condensation.

With the fermions in liquid ${ }^{3} \mathrm{He}$ able to form boson pairs, it was expected that superfluidity would be obtainable at very low temperatures. After much work many felt that superfluidity in ${ }^{3} \mathrm{He}$ was impossible to achieve, until its discovery in 1971 by this year's Nobel laureates at temperatures around $2 \mathrm{mK}$. Studying this exotic

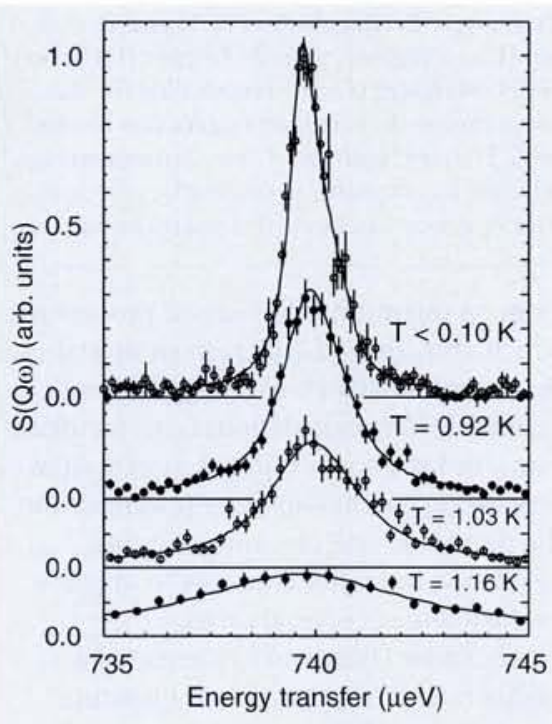

Fig. 2. Roton peaks measured on the ILL's IN10B spectrometer at four different temperatures. The solid line is the best-fit line shape. The shoulder on the high-energy side, which is most clearly seen at low temperature, is caused by the width of the instrumental Q-resolution.

Fig. 3. Measured roton parameters. a: The best-fit halfwidth compared with the 1984 neutron spin echo data of Mezei and the values predicted by Bedell et al. The agreement between experiment and theory is excellent. b: Best-fit roton energy: the observed temperature dependence is clearly less than that expected from the theory of Bedell et al. quantum liquid and its rich set of physical properties has since led to concepts of general importance which could, for example, help in understanding hightemperature superconductors.

Despite its apparent simplicity, pure liquid ${ }^{4} \mathrm{He}$ constitutes a fascinatingly complex many-body system and still provides intriguing and challenging work for theorists and experimentalists alike. Neutron scattering measurements which probe the density fluctuations of a system show that the elementary excitations in superfluid ${ }^{4} \mathrm{He}$ follow a dispersion curve known as the phonon - roton curve (Fig. 1). Superfluidity can be inferred from the kinematical restraints on creating an elementary excitation, and it can be shown [1] that for low flow velocities, the only contributions to the viscosity arise from the thermally excited elementary excitations; the actual

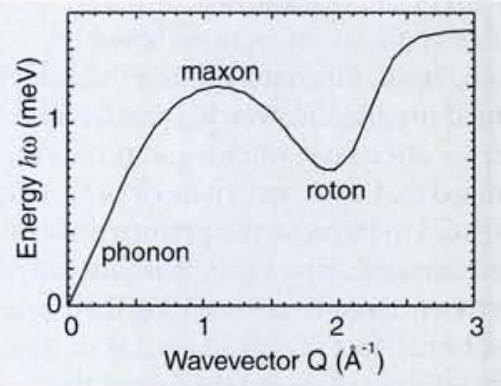

Fig. 1. Phonon-roton dispersion curve of superfluid ${ }^{4} \mathrm{He}$.
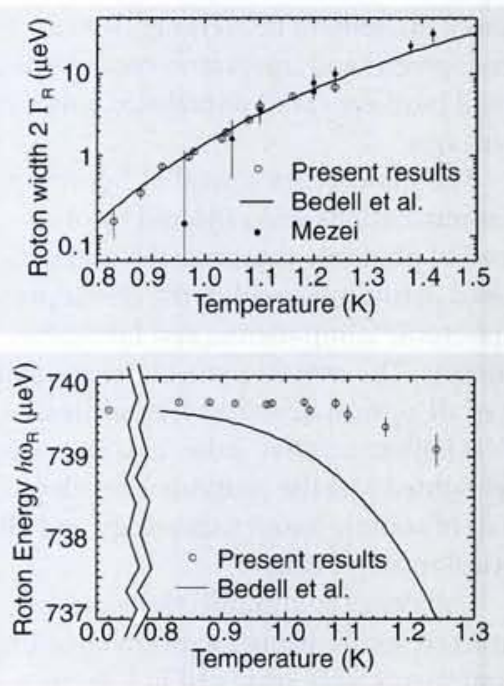